\title{
Certain fractional formulas of the extended k-hypergeometric functions
}

\author{
Mohamed Abdalla ${ }^{1,2}$, Salah Boulaaras ${ }^{3,4^{*}}$, Mohamed Akel ${ }^{2}$, Sahar Ahmed Idris ${ }^{5}$ and Shilpi Jain ${ }^{6}$
}

\section{"Correspondence:}

s.boularas@qu.edu.sa

${ }^{3}$ Department of Mathematics,

College of Sciences and Arts, ArRas,

Qassim University, Buraydah,

Kingdom of Saudi Arabia

${ }^{4}$ Laboratory of Fundamental and

Applied Mathematics of Oran

(LMFAO), University of Oran 1, Oran, Algeria

Full list of author information is available at the end of the article

\begin{abstract}
In this article, we aim to investigate various formulae for the $(p, k)$-analogues of Gauss hypergeometric functions, including the integral transforms and the operators of fractional calculus. All the outcomes presented here are of general attractiveness and can yield a number of previous works as special cases.
\end{abstract}

MSC: 33B15; 33C20; 33C90; 33E20

Keywords: $(p, k)$-hypergeometric functions; Integral transforms; Fractional calculus

\section{Overture}

Throughout this work, $\mathbb{N}:=\{1,2,3, \ldots\}$ denotes the set of positive integers, $\mathbb{N}_{0}=\mathbb{N} \cup\{0\}$, $\mathbb{Z}^{-}:=\{-1,-2,-3, \ldots\}$ denotes the set of negative integers, $\mathbb{Z}_{0}^{-}=\mathbb{Z}^{-} \cup\{0\}, \mathbb{R}^{+}$denotes the set of positive real numbers, and $\mathbb{C}$ denotes the set of complex numbers.

In 1813, Gauss first summarized his investigations of hypergeometric functions, which has been of great significance in the mathematical modeling of physical phenomena and other applications. Recently, various developments and expansions of the hypergeometric functions have been proposed and discussed (for example, see [1-12]).

In [13], Diaz and Pariguan introduced an interesting extensions of the gamma, beta, Pochhammer, and hypergeometric functions as follows.

Definition 1.1 For $k \in \mathbb{R}^{+}$, the k-gamma function $\Gamma^{k}(y)$ is defined by

$$
\Gamma^{k}(y)=\int_{0}^{\infty} u^{y-1} e^{-\frac{u^{k}}{k}} d u
$$

where $y \in \mathbb{C} \backslash k \mathbb{Z}^{-}$. We note that $\Gamma^{k}(y) \rightarrow \Gamma(y)$ for $k \rightarrow 1$ where $\Gamma(y)$ is the classical Euler's gamma function and $(y)_{m, k}$ is the k-Pochhammer symbol given by

$$
(y)_{n, k}=\frac{\Gamma^{k}(y+n k)}{\Gamma^{k}(y)}= \begin{cases}y(y+k) \ldots(y+(n-1) k), & n \in \mathbb{N}, y \in \mathbb{C}, \\ 1, & n=0, k \in \mathbb{R}^{+}, y \in \mathbb{C} \backslash\{0\},\end{cases}
$$

\section{Springer}

(c) The Author(s) 2021. This article is licensed under a Creative Commons Attribution 4.0 International License, which permits use, sharing, adaptation, distribution and reproduction in any medium or format, as long as you give appropriate credit to the original author(s) and the source, provide a link to the Creative Commons licence, and indicate if changes were made. The images or other third party material in this article are included in the article's Creative Commons licence, unless indicated otherwise in a credit line to the material. If material is not included in the article's Creative Commons licence and your intended use is not permitted by statutory regulation or exceeds the permitted use, you will need to obtain permission directly from the copyright holder. To view a copy of this licence, visit http://creativecommons.org/licenses/by/4.0/. 
the relation between the $\Gamma^{k}(y)$ and the usual gamma function $\Gamma(y)$ follows easily as

$$
\Gamma^{k}(y)=k^{\frac{y}{k}-1} \Gamma\left(\frac{y}{k}\right) \quad \text { or } \quad \Gamma(w)=k^{1-w} \Gamma^{k}(k w)
$$

Definition 1.2 The k-beta function $\mathbf{B}^{k}(s, t)$ is defined by

$$
\mathbf{B}^{k}(s, t)= \begin{cases}\frac{1}{k} \int_{0}^{1} y^{\frac{s}{k}-1}(1-y)^{\frac{t}{k}-1} d y, & \left(k \in \mathbb{R}^{+}, \min \{\operatorname{Re}(s), \operatorname{Re}(t)\}>0\right), \\ \frac{\Gamma^{k}(s) \Gamma^{k}(t)}{\Gamma^{k}(s+t)}, & \left(k \in \mathbb{R}^{+}, s, t \in \mathbb{C} \backslash \mathbb{Z}_{0}^{-}\right) .\end{cases}
$$

Clearly, the case $k=1$ in (1.3) reduces to the known beta function $\mathbb{B}(s, t)$.

$$
\mathbb{B}(s, t)=\int 1_{0} y^{s-1}(1-y)^{t-1} d y .
$$

Also, the relation between the k-beta function $\mathbf{B}^{k}(s, t)$ and the original beta function $\mathbb{B}(s, t)$ is

$$
\mathbf{B}^{k}(s, t)=\frac{1}{k} \mathbb{B}\left(\frac{s}{k}, \frac{t}{k}\right)
$$

Definition 1.3 Let $k \in \mathbb{R}^{+}$and $\alpha_{1}, \alpha_{2}, y \in \mathbb{C}$ and $\alpha_{3} \in \mathbb{C} \backslash \mathbb{Z}_{0}^{-}$, then k-hypergeometric series is defined by the form

$$
{ }_{2} H_{1}^{k}\left[\begin{array}{c}
\left(\alpha_{1} ; k\right),\left(\alpha_{2} ; k\right) \\
\left(\alpha_{3} ; k\right) ;
\end{array}\right]=\sum_{n=0}^{\infty} \frac{\left(\alpha_{1}\right)_{n, k}\left(\alpha_{2}\right)_{n, k}}{\left(\alpha_{3}\right)_{n, k}} \cdot \frac{y^{n}}{n !}, \quad|y|<\frac{1}{k},
$$

where $\left(\alpha_{1}\right)_{n, k}$ is the k-Pochhammer symbol given in (1.2).

Indeed, in their special case when $k=1$, Eq. (1.4) is reduced to the Gauss hypergeometric function ${ }_{2} H_{1}(\cdot)$. The ${ }_{2} H_{1}(\cdot)$ is the special case of the generalized hypergeometric functions ${ }_{m} H_{n}(\cdot)$ of $m$ numerator and $n$ denominator parameters defined by (see, e.g., [14, Sect. 1.5$]$ ):

$$
{ }_{m} H_{n}\left[\begin{array}{c}
\alpha_{1} \ldots \alpha_{m} ; \\
\delta_{1} \ldots \delta_{n} ;
\end{array}\right]=\sum_{j=0}^{\infty} \frac{\left(\alpha_{1}\right)_{j} \ldots\left(\alpha_{m}\right)_{j}}{\left(\delta_{1}\right)_{j} \ldots\left(\delta_{n}\right)_{j}} \cdot \frac{y^{j}}{j !}
$$

where

$$
\left(\alpha_{1}\right)_{n}=\frac{\Gamma\left(\alpha_{1}+n\right)}{\Gamma\left(\alpha_{1}\right)}= \begin{cases}\alpha_{1}\left(\alpha_{1}+1\right) \ldots\left(\alpha_{1}+n-1\right), & i \in \mathbb{N}, \alpha_{1} \in \mathbb{C}, \\ 1, & i=0 ; \alpha_{1} \in \mathbb{C} \backslash\{0\}\end{cases}
$$

is the usual Pochhammer symbol (or the shifted factorial) and $\Gamma(\cdot)$ is the standard gamma function (see, e.g., [14, Sect. 1.1])).

Currently, several different outcomes concerning the k-analogue of special functions have been archived, the interested reader may refer to the monographs by many researchers (see, e.g., [15-22] and the references cited therein). 
Recently, Abdalla and Hidan [23] and Hidan et al. [24] introduced and studied several properties of the following $(p, k)$-analogues of Gauss hypergeometric functions:

$$
{ }_{2} \mathbf{H}_{1}^{(p, k)}\left[\begin{array}{c}
\left(\alpha_{1} ; k\right),\left(\alpha_{2} ; k\right) \\
\left(\alpha_{3} ; k\right)
\end{array} ; w=\sum_{j=0}^{\infty} \frac{\left(\alpha_{1}\right)_{j, k}\left(\alpha_{2}\right)_{j, k}}{\left(\alpha_{3}\right)_{j, k}} \cdot \frac{w^{j}}{(p j) !},\right.
$$

which is an entire function for $p>1$, where $k \in \mathbb{R}^{+}$and $\alpha_{1}, \alpha_{2}, w \in \mathbb{C}$ and $\alpha_{3} \in \mathbb{C} \backslash \mathbb{Z}_{0}^{-}$, and $(\alpha)_{j, k}$ is the k-Pochhammer symbol defined in (1.2).

Remark 1.1 Among the important special cases of ${ }_{2} \mathbf{H}_{1}^{(p, k)}$ are equations of type (1.4) and (1.5). Further, at $k=1$, we obtain the $p$-extended Gauss hypergeometric functions in the following form (cf. [25]):

$$
{ }_{2} \mathbf{H}_{1}^{(p)}\left[\begin{array}{c}
\alpha_{1}, \alpha_{2} \\
\alpha_{3}
\end{array} ; w\right]=\sum_{j=0}^{\infty} \frac{\left(\alpha_{1}\right)_{j}\left(\alpha_{2}\right)_{j}}{\left(\alpha_{3}\right)_{j}} \cdot \frac{w^{j}}{(p j) !}, \quad p \in \mathbb{N},
$$

which is also an entire function for $p>1$.

The purpose of this work is to continue the investigation of new formulae like integral transforms and fractional calculus operators on the $(p, k)$-analogues of Gauss hypergeometric functions ${ }_{2} \mathbf{H}_{1}^{(p, k)}$. In Sects. 2 and 3, respectively, we introduce several integral transforms and image formulae for the $(p, k)$-analogues of Gauss hypergeometric functions ${ }_{2} \mathbf{H}_{1}^{(p, k)}$ by applying a certain integral transform (like Laplace transform and fractional Fourier transform) and diverse fractional operators. Also, some special cases and significance of our main outcomes are considered.

\section{Integral transforms}

In this section, we prove two theorems, which exhibit the connection between integral transforms like the Laplace transform and the fractional Fourier transform for the ${ }_{2} \mathbf{H}_{1}^{(p, k)}$ given in (1.7). We recall the Laplace transform and the fractional Fourier transform, respectively.

Definition 2.1 (Laplace transform) Let $f(\xi)$ be a function of $\xi>0$. Then the Laplace transform of $f(\xi)$ is defined by

$$
\mathbf{F}(s)=\mathcal{L}\{f(\xi): s\}=\int_{0}^{\infty} e^{-s \xi} f(\xi) d \xi, \quad \Re(s)>0,
$$

provided that the improper integral exists, $e^{-\lambda \xi}$ is the kernel of the transformation, and the function $f(\xi)$ is called the inverse Laplace transform of $\mathbf{F}(\lambda)$ (see [26, Chap. 3]).

Definition 2.2 (Fractional Fourier transform) Assume that $\varphi$ is a function belonging to Lizorkin space $\psi(R)$. The fractional Fourier transform (FFT) of order $\beta, 0<\beta \leq 1$, is defined as (cf. [26, 27])

$$
\varphi_{\beta}(\omega)=\mathfrak{F}_{\beta}[\varphi](\omega)=\int_{R} e^{i \omega^{\frac{1}{\beta}} \xi} \varphi(\xi) d \xi, \quad i=\sqrt{-1}
$$


Remark 2.1 It may by observed that when $\omega>0$ it reduces to the FFT introduced by Luchko, Martinez, and Trujillo (see, e.g., [27, pp. 225-240] for details). The relationship between the two Fourier transforms, the classical and FFT, is given by the following relation:

$$
\mathfrak{F}_{\beta}[\varphi](\omega)=\mathfrak{F}[\varphi](s) \quad \text { for } s=\omega^{\frac{1}{\beta}}, 0<\beta \leq 1
$$

Theorem 2.1 The Laplace transform for the ${ }_{2} \mathbf{H}_{1}^{(p, k)}$ given by (1.7) is in the form

$$
\begin{aligned}
& \mathcal{L}\left\{\xi^{\frac{\delta}{k}-1}{ }_{2} \mathbf{H}_{1}^{(p, k)}\left[\begin{array}{cc}
\left(\alpha_{1}, k\right)\left(\alpha_{2}, k\right) & u \xi \\
\left(\alpha_{3}, k\right) ; &
\end{array}\right]\right\} \\
& =\frac{k \Gamma^{k}(\delta)}{(k s)^{\frac{\delta}{k}}}{ }_{3} \mathbf{H}_{1}^{(p, k)}\left[\begin{array}{cc}
\left(\alpha_{1}, k\right)\left(\alpha_{2}, k\right)(\delta, k) & u \\
\left(\alpha_{3}, k\right) ; & k s
\end{array}\right],
\end{aligned}
$$

$\left(\alpha_{1}, \alpha_{2}, u, \xi \in \mathbb{C}, \alpha_{3} \in \mathbb{C} \backslash \mathbb{Z}_{0}^{-}, \operatorname{Re}\left(\alpha_{1}\right)>0, \operatorname{Re}\left(\alpha_{2}\right)>0, \operatorname{Re}(s)>0,\left|\frac{u}{k s}\right|<1, k \in \mathbb{R}^{+}\right.$and $\left.p \in \mathbb{N}\right)$

Proof Taking the left-hand side of Eq. (2.3) by $\mathfrak{I}$ and upon using (1.7), we have

$$
\begin{aligned}
\mathfrak{I} & =\int_{0}^{\infty} e^{-s \xi} \xi^{\frac{\delta}{k}-1} \sum_{n=0}^{\infty} \frac{\left(\alpha_{1}\right)_{n, k}\left(\alpha_{2}\right)_{n, k}(u \xi)^{n}}{\left(\alpha_{3}\right)_{n, k}(p n) !} d \xi \\
& =\sum_{n=0}^{\infty} \frac{\left(\alpha_{1}\right)_{n, k}\left(\alpha_{2}\right)_{n, k}}{\left(\alpha_{3}\right)_{n, k}} \frac{u^{n}}{(p n) !}\left\{\int_{0}^{\infty} e^{-s \xi} \xi^{\frac{\delta}{k}+n-1} d \xi\right\} .
\end{aligned}
$$

Putting $s \xi=\frac{v^{k}}{k}$, we have

$$
\begin{aligned}
\mathfrak{I} & =\sum_{n=0}^{\infty} \frac{\left(\alpha_{1}\right)_{n, k}\left(\alpha_{2}\right)_{n, k}}{\left(\alpha_{3}\right)_{n, k}} \frac{u^{n}}{(n p) !} \int_{0}^{\infty} e^{\frac{-v^{k}}{k}}\left(\frac{v^{k}}{k s}\right)^{\frac{\delta}{k}+n-1} \frac{v^{k-1}}{s} d v \\
& =\sum_{n=0}^{\infty} \frac{\left(\alpha_{1}\right)_{n, k}\left(\alpha_{2}\right)_{n, k}}{\left(\alpha_{3}\right)_{n, k}} \frac{u^{n}}{(n p) !} \int_{0}^{\infty} \frac{e^{\frac{-v^{k}}{k}} v^{\delta+n k-1}}{k^{\frac{\delta}{k}+n-1} s^{\frac{\delta}{k}+n}} \\
& =\frac{k \Gamma^{k}(\delta)}{(k s)^{\frac{\delta}{k}}} \sum_{n=0}^{\infty}\left(\frac{u}{k s}\right)^{n} \frac{\left(\alpha_{1}\right)_{n, k}\left(\alpha_{2}\right)_{n, k}(\delta)_{n, k}}{\left(\alpha_{3}\right)_{n, k}} \frac{1}{(p n) !} .
\end{aligned}
$$

We thus obtain the required result.

Theorem 2.2 For $\alpha_{1}, \alpha_{2}, w \in \mathbb{C}, \alpha_{3} \in \mathbb{C} \backslash \mathbb{Z}_{0}^{-}, \operatorname{Re}\left(\alpha_{1}\right)>0, \operatorname{Re}\left(\alpha_{2}\right)>0, k \in \mathbb{R}^{+}, p \in \mathbb{N}$, and $0<\beta \leq 1$, the following fractional Fourier transform (FFT) holds true:

$$
\begin{aligned}
\mathfrak{F}_{\beta} & \left\{{ }_{2} \mathbf{H}_{1}^{(p, k)}\left[\begin{array}{c}
\left(\alpha_{1}, k\right)\left(\alpha_{2}, k\right) \\
\left(\alpha_{3}, k\right)
\end{array} ; w\right]\right) \\
= & \sum_{n=0}^{\infty} \frac{\left(\alpha_{1}\right)_{n, k}\left(\alpha_{2}\right)_{n, k}}{\left(\alpha_{3}\right)_{n, k}}(\omega)^{-\left(\frac{n+1}{\beta}\right)}(-1)^{n}(i)^{(n-1)} \\
& \times \frac{n !}{(p n) !}
\end{aligned}
$$


Proof For convenience, let the left-hand side of (2.4) be denoted by T. Applying the fractional Fourier transform (2.2) to (1.7) when $w<0$, we observe that

$$
\begin{aligned}
\mathrm{T} & =\int_{R} e^{i \omega^{\frac{1}{\beta}} w} \sum_{n=0}^{\infty} \frac{\left(\alpha_{1}\right)_{n, k}\left(\alpha_{2}\right)_{n, k}}{\left(\alpha_{3}\right)_{n, k}(n p) !} w^{n} d w \\
& =\sum_{n=0}^{\infty} \frac{\left(\alpha_{1}\right)_{n, k}\left(\alpha_{2}\right)_{n, k}}{\left(\alpha_{3}\right)_{n, k}} \int_{-\infty}^{0} e^{i \omega^{\frac{1}{\beta}} w} w^{n} d w .
\end{aligned}
$$

Letting $-t=i \omega^{\frac{1}{\beta}} w$, we obtain

$$
\begin{aligned}
\mathrm{T} & =\sum_{n=0}^{\infty} \frac{\left(\alpha_{1}\right)_{n, k}\left(\alpha_{2}\right)_{n, k}}{\left(\alpha_{3}\right)_{n, k}(n p) !} \int_{0}^{\infty} e^{-t}\left(\frac{-t}{i \omega^{\frac{1}{\beta}}}\right)^{n}\left(\frac{d t}{i \omega^{\frac{1}{\beta}}}\right) \\
& =\sum_{n=0}^{\infty} \frac{\left(\alpha_{1}\right)_{n, k}\left(\alpha_{2}\right)_{n, k}}{\left(\alpha_{3}\right)_{n, k}(n p) !}(-1)^{n}(i)^{-(n+1)}(\omega)^{\frac{-(n+1)}{\beta}} \Gamma(n+1),
\end{aligned}
$$

which yields our required result (2.4).

Corollary 2.1 For $p=1$, the FFT of $k$-Gauss hypergeometric function of order $\beta$ is (see [28])

$$
\mathfrak{F}_{\beta}\left[{ }_{2} \mathbf{H}_{1}^{(k)}\left[\begin{array}{c}
\left(\alpha_{1}, k\right)\left(\alpha_{2}, k\right) \\
\left(\alpha_{3}, k\right)
\end{array} ; w\right]\right)=\sum_{n=0}^{\infty} \frac{\left(\alpha_{1}\right)_{n, k}\left(\alpha_{2}\right)_{n, k}}{\left(\alpha_{3}\right)_{n, k}}(-1)^{n}(i)^{-(n+1)}(\omega)^{\frac{-(n+1)}{\beta}} .
$$

Further, for $p=1$ and $k=1$, we get the FFT of Gauss hypergeometric function

$$
\mathfrak{F}_{\beta}\left[{ }_{2} \mathbf{H}_{1}\left[\begin{array}{c}
\left(\alpha_{1}\right)\left(\alpha_{2}\right) \\
\left(\alpha_{3}\right)
\end{array} ; w\right]\right)=\sum_{n=0}^{\infty} \frac{\left(\alpha_{1}\right)_{n}\left(\alpha_{2}\right)_{n}}{\left(\alpha_{3}\right)_{n}}(-1)^{n}(i)^{-(n+1)}(\omega)^{\frac{-(n+1)}{\beta}} .
$$

\section{3 k-fraction calculus of the ${ }_{2} \mathrm{H}_{1}^{(p, k)}$}

Nowadays, computations of images of k-analogues of special functions under operators of $\mathrm{k}$-fractional calculus have found significant importance and applications by many references (for instance, see [15-17, 28-40]).

The k-Riemann-Liouville fractional integral using k-gamma function is defined in [31] as follows:

$$
\left(\mathbf{I}_{k}^{v} f(\tau)\right)(x)=\frac{1}{k \Gamma^{k}(v)} \int_{0}^{x} f(\tau)(x-\tau)^{\frac{v}{k}-1} d \tau, \quad v, k \in \mathbb{R}^{+}
$$

Therefore, the k-Riemann-Liouville fractional derivative of order $v$ is introduced in [29, 31] by

$$
\mathbf{D}_{k}^{v}\{f(\eta)\}=D\left(\mathbf{I}_{k}^{(1-v)} f(\eta)\right) ; \quad 0<v \leq 1, D=\frac{d}{d \eta}
$$


Let $\alpha, \beta, \gamma, \delta, \eta \in \mathbb{C}(\operatorname{Re}(\eta)>0)$ and $x>0$, then the generalized fractional calculus operator (the Marichev-Saigo-Maeda operator) is defined by (see [33, 41, 42])

$$
\begin{aligned}
\left(\mathbf{I}_{0, x}^{\alpha, \beta, \gamma, \delta, \eta}\right) f(x)= & \frac{x^{-\alpha}}{\Gamma(\eta)} \int_{0}^{x}(x-t)^{\eta-1} t^{-\beta} \\
& \times F_{3}\left[\begin{array}{cccc}
\alpha & \beta & \gamma & \delta \\
\eta & & ; 1-\frac{t}{x}, 1-\frac{x}{t}
\end{array}\right] f(t) d t,
\end{aligned}
$$

where $F_{3}$ denotes the Appell third function, known also as Horn's $F_{3}$-function for $(\max \{|z|<1,|w|\}<1)$ defined by the series

$$
F_{3}\left[\begin{array}{cccc}
\alpha & \beta & \gamma & \delta \\
& \eta & & ; z, w
\end{array}\right]=\sum_{m, n=0}^{\infty} \frac{(\alpha)_{m}(\beta)_{n}(\gamma)_{m}(\delta)_{n}}{(\eta)_{m+n} m ! n !} z^{m} w^{n}
$$

which reduces to the Gauss hypergeometric function as follows:

$$
\begin{aligned}
{ }_{2} \mathbf{H}_{1}\left[\begin{array}{c}
\alpha, \beta \\
\eta
\end{array} ;\right] & =F\left[\begin{array}{cccc}
\alpha & \beta & \gamma & \delta \\
& \eta & & ; z, 0
\end{array}\right]=F_{3}\left[\begin{array}{cccc}
\alpha & 0 & \gamma & \delta \\
& \eta & & ; z, w
\end{array}\right] \\
& =F_{3}\left[\begin{array}{cccc}
\alpha & \beta & \gamma & 0 \\
& \eta & & ; z, w
\end{array}\right] .
\end{aligned}
$$

The following image formula, which is required in the sequel, can be easily derived from the direct application of the fractional integral operator (3.3), (see, e.g., [41, 42]):

$$
\begin{aligned}
& \left(\mathbf{I}_{0, x}^{\alpha, \beta, \gamma, \delta, \eta} t^{\theta-1}\right)(x) \\
& \quad=\frac{\Gamma(\theta) \Gamma(\theta+\eta-\alpha-\beta-\gamma) \Gamma(\theta+\delta-\beta)}{\Gamma(\theta+\delta) \Gamma(\theta+\eta-\alpha-\beta) \Gamma(\theta+\eta-\beta-\gamma)} x^{\theta+\eta-\alpha-\beta-1},
\end{aligned}
$$

where $\operatorname{Re}(\eta)>0, \operatorname{Re}(\theta)>\max \{0, \operatorname{Re}(\alpha+\beta+\gamma-\eta), \operatorname{Re}(\beta-\delta)\}$.

Here, we aim at establishing certain new image formulas for the $(p, k)$-analogues of Gauss hypergeometric functions by applying the k-fractional derivative by (3.2) and leftsided operator of Marichev-Saigo-Maeda fractional integral defined by (3.3). On account of the general nature of the hypergeometric functions, a number of known formulas can easily be found as special cases of our main outcomes.

Theorem 3.1 For $\alpha_{1}, \alpha_{2}, v, u \in \mathbb{C}, \alpha_{3} \in \mathbb{C} \backslash \mathbb{Z}_{0}^{-}, \operatorname{Re}\left(\alpha_{1}\right)>0, \operatorname{Re}\left(\alpha_{2}\right)>0, k \in \mathbb{R}^{+}, p \in \mathbb{N}$, and $0<\Re(v) \leq 1$, we have

$$
\begin{aligned}
\mathbf{D}_{k}^{v} & \left\{u^{\frac{\delta}{k}}{ }_{2} \mathbf{H}_{1}^{(p, k)}\left[\begin{array}{c}
\left(\alpha_{1}, k\right)\left(\alpha_{2}, k\right) \\
\left(\alpha_{3}, k\right)
\end{array} ; u\right\}\right. \\
& =\frac{\lambda \Gamma^{k}(\lambda)}{k \Gamma^{k}(1-v+\delta)} u^{\frac{1-v+\delta}{k}-1}{ }_{3} \mathbf{H}_{2}^{(p, k)}\left[\begin{array}{c}
\left(\alpha_{1}, k\right)\left(\alpha_{2}, k\right)(\delta+k, k) \\
\left(\alpha_{3}, k\right)(1-v+\delta, k)
\end{array} ; u .\right.
\end{aligned}
$$


Proof From (1.7) and (3.2), we observe that

$$
\begin{aligned}
\mathbf{D}_{k}^{v} & {\left[u^{\frac{\delta}{k}}{ }_{2} \mathbf{H}_{1}^{(p, k)}\left[\begin{array}{c}
\left(\alpha_{1}, k\right)\left(\alpha_{2}, k\right) \\
\left(\alpha_{3}, k\right)
\end{array} ; u\right]\right] } \\
& =\frac{d}{d u}\left[\mathbf{I}_{k}^{1-v} u^{\frac{\delta}{k}}{ }_{2} \mathbf{H}_{1}^{(p, k)}\left[\begin{array}{c}
\left(\alpha_{1}, k\right)\left(\alpha_{2}, k\right) \\
\left(\alpha_{3}, k\right)
\end{array} ;\right]\right] d t \\
& \left.=\frac{d}{d u} \frac{1}{k \Gamma^{k}(1-v)} \int_{0}^{u}(u-t)^{\frac{1-v}{k}-1} t^{\frac{\delta}{k}}{ }_{2} \mathbf{H}_{1}^{(p, k)}\left[\begin{array}{c}
\left(\alpha_{1}, k\right)\left(\alpha_{2}, k\right) \\
\left(\alpha_{3}, k\right)
\end{array}\right]\right] d t .
\end{aligned}
$$

Putting $t=u x$ in the above equation and after simple computations, we arrive at

$$
\begin{aligned}
& \mathbf{D}_{k}^{v}\left[u^{\frac{\delta}{k}}{ }_{2} \mathbf{H}_{1}^{(p, k)}\left[\begin{array}{c}
\left(\alpha_{1}, k\right)\left(\alpha_{2}, k\right) \\
\left(\alpha_{3}, k\right)
\end{array}\right]\right] \\
& =\frac{1}{k \Gamma^{k}(1-v)} \sum_{n=0}^{\infty} \frac{\left(\alpha_{1}\right)_{n, k}\left(\alpha_{2}\right)_{n, k}}{\left(\alpha_{3}\right)_{n, k}(n p) !} \frac{d}{d u} \int_{0}^{1}(u-u x)^{\frac{1-v}{k}-1}(u x)^{n+\frac{\delta}{k}} u d x \\
& =\frac{1}{k \Gamma^{k}(1-v)} \sum_{n=0}^{\infty} \frac{\left(\alpha_{1}\right)_{n, k}\left(\alpha_{2}\right)_{n, k}}{\left(\alpha_{3}\right)_{n, k}(n p) !} \frac{d}{d u} \int_{0}^{1}(1-x)^{\frac{1-v}{k}-1}(x)^{n+\frac{\delta}{k}} u^{\frac{1-v+\delta+n k}{k}} d x \\
& =\frac{1}{k \Gamma^{k}(1-v)} \sum_{n=0}^{\infty} \frac{\left(\alpha_{1}\right)_{n, k}\left(\alpha_{2}\right)_{n, k}}{\left(\alpha_{3}\right)_{n, k}(n p) !} \frac{d}{d u} u^{\frac{1-v+\delta+n k}{k}} \mathbb{B}\left(\frac{1-v}{k}, n+\frac{\delta}{k}+1\right) \\
& =\frac{1}{k \Gamma^{k}(1-v)} \sum_{n=0}^{\infty}\left[\frac{\left(\alpha_{1}\right)_{n, k}\left(\alpha_{2}\right)_{n, k}}{\left(\alpha_{3}\right)_{n, k}(n p) !}\left(\frac{1-v+\delta+n k}{k}\right) u^{\frac{1-v+\delta+n k}{k}}-1 \frac{\Gamma\left(\frac{1-v}{k}\right) \Gamma\left(\frac{n k+\delta+k}{k}\right)}{\left(\frac{1-v+\lambda+n k+}{k}\right)}\right] \\
& =\sum_{n=0}^{\infty} \frac{\left(\alpha_{1}\right)_{n, k}\left(\alpha_{2}\right)_{n, k}}{\left(\alpha_{3}\right)_{n, k}} \frac{\Gamma^{k}(n k+\delta+k)}{k \Gamma^{k}(1-v+\delta+n k)} \frac{u^{\frac{1-v+\delta+n k}{k}-1}}{(n p) !} \\
& =u^{\frac{1-v+\delta}{k}-1} \frac{\delta \Gamma^{k}(\delta)}{k \Gamma^{k}(1-v+\delta)} \sum_{n=0}^{\infty} \frac{\left(\alpha_{1}\right)_{n, k}\left(\alpha_{2}\right)_{n, k}(\delta+k)_{n, k}}{\left(\alpha_{3}\right)_{n, k}(1-v+\delta)_{n, k}} \frac{u^{n}}{(n p) !} .
\end{aligned}
$$

This completes the proof of Theorem 3.1.

Theorem 3.2 Assume that $\alpha, \beta, \gamma, \delta, \eta, \vartheta, \alpha_{1}, \alpha_{2} \in \mathbb{C}, \alpha_{3} \in \mathbb{C} \backslash \mathbb{Z}_{0}^{-}, x>0, k \in \mathbb{R}^{+}$, and $p \in \mathbb{N}$ such that $\mathfrak{R}\left(\frac{\vartheta}{k}\right)>\max \{0, \mathfrak{R}(\beta-\delta), \mathfrak{R}(\alpha+\beta+\gamma-\eta)\}$, then we have

$$
\begin{aligned}
& \left(\mathbf{I}_{0, x}^{\alpha, \beta, \gamma, \delta, \eta} w^{\frac{\vartheta}{k}-1}{ }_{2} \mathbf{H}_{1}^{(p, k)}\left[\begin{array}{c}
\left(\alpha_{1} ; k\right),\left(\alpha_{2} ; k\right) \\
\left(\alpha_{3} ; k\right)
\end{array}\right]\right)(x) \\
& =k^{\eta} x^{-\alpha-\beta+\eta+\frac{\vartheta}{k}-1} \frac{\Gamma^{k}(\vartheta) \Gamma^{k}(\vartheta-k \beta+k \delta) \Gamma^{k}(\vartheta-k \alpha-k \beta-k \gamma+k \eta)}{\Gamma^{k}(\vartheta+k \delta) \Gamma^{k}(\vartheta-k \alpha-k \beta+k \eta) \Gamma^{k}(\vartheta-k \beta-k \gamma+k \eta)} \\
& \times{ }_{5} \mathbf{H}_{4}^{(p, k)}\left[\begin{array}{ccccc}
\left(\alpha_{1} ; k\right) & \left(\alpha_{2} ; k\right) & (\vartheta ; k) & (\vartheta-k \beta+k \delta ; k) & (\vartheta-k \alpha-k \beta-k \gamma+k \eta ; k) \\
& \left(\alpha_{3} ; k\right) & (\vartheta+k \delta ; k) & (\vartheta-k \alpha-k \beta+k \eta ; k) & (\vartheta-k \beta-k \gamma+k \eta ; k)
\end{array}\right] .
\end{aligned}
$$


Proof We indicate the left-hand side of(3.6) by $\Upsilon$, and invoking to Eqs. (3.3) and (1.7), we find

$$
\begin{aligned}
\Upsilon= & \left(\mathbf{I}_{0, x}^{\alpha, \beta, \gamma, \delta, \eta} w^{\frac{\vartheta}{k}-1} \sum_{n=0}^{\infty} \frac{\left(\alpha_{1}\right)_{n, k}\left(\alpha_{2}\right)_{n, k}}{\left(\alpha_{3}\right)_{n, k}} \frac{w^{n}}{(p n) !}\right)(x) \\
= & \sum_{n=0}^{\infty} \frac{\left(\alpha_{1}\right)_{n, k}\left(\alpha_{2}\right)_{n, k}}{\left(\alpha_{3}\right)_{n, k}} \frac{1}{(p n) !}\left(\mathbf{I}_{0, x}^{\alpha, \beta, \gamma, \delta, \eta} w^{\frac{\vartheta}{k}+n-1}\right)(x) \\
= & \sum_{n=0}^{\infty} \frac{\left(\alpha_{1}\right)_{n, k}\left(\alpha_{2}\right)_{n, k}}{\left(\alpha_{3}\right)_{n, k}} \frac{\Gamma\left(\frac{\vartheta}{k}+n\right)}{(p n) ! \Gamma\left(\delta+\frac{\vartheta}{k}+n\right)} \\
& \times \frac{\Gamma\left(-\beta+\delta+\frac{\vartheta+n k}{k}\right) \Gamma\left(-\alpha-\beta-\gamma+\eta+\frac{\vartheta+n k}{k}\right)}{\Gamma\left(-\alpha-\beta+\eta+\frac{\vartheta+n k}{k}\right) \Gamma\left(-\beta-\gamma+\eta+\frac{\vartheta+n k}{k}\right)} x^{-\alpha-\beta+\eta+\frac{\vartheta+n k}{k}-1} .
\end{aligned}
$$

Upon using (3.4) and after a simplification, we get the following expression:

$$
\begin{aligned}
\Upsilon= & \sum_{n=0}^{\infty} \frac{\left(\alpha_{1}\right)_{n, k}\left(\alpha_{2}\right)_{n, k}}{\left(\alpha_{3}\right)_{n, k}} \frac{x^{n} k^{\eta}}{(p n) !} \frac{(\vartheta)_{n, k} \Gamma^{k}(\vartheta)}{(\vartheta+k \delta)_{n, k} \Gamma^{k}(\vartheta+k \delta)} \\
& \times \frac{(\vartheta-k \beta+k \delta)_{n, k} \Gamma^{k}(-k \beta+k \delta+\vartheta)}{(\vartheta-k \alpha-k \beta+k \eta)_{n, k} \Gamma^{k}(\vartheta-k \alpha-k \beta+k \eta)} \\
& \times \frac{(\vartheta-k \alpha-k \beta+k \eta)_{n, k}}{(\vartheta-k \beta-k \gamma+k \eta)_{n, k}} \cdot \frac{\Gamma^{k}(\vartheta-k \alpha-k \beta-k \gamma+k \eta)}{\Gamma^{k}(\vartheta-k \beta-k \gamma+k \eta)} x^{-\alpha-\beta+\eta+\frac{\vartheta}{k}-1},
\end{aligned}
$$

whose last summation, in view of (1.2), is easily seen to arrive at the expression in (3.6). This completes the proof of Theorem 3.2.

\section{Conclusion}

Recently, the applications and importance of integral transforms and fractional calculus operators involving a variety of special functions have received more attention in various fields like mathematical analysis, survival analysis, physics, statistics, and engineering. In fact, this manuscript is a continuation of the recent authors' articles [23, 24], where we have introduced the $(p, k)$-analogues of hypergeometric functions and their various properties and applications. In this line of research, we have derived integral transforms and image formulas for the $(p, k)$-analogues of hypergeometric functions. We also have considered that by setting $p \rightarrow 1$, the various outcomes considered in this manuscript reduce to the corresponding outcomes (see $[28,31,36]$ ). Also, for $k \rightarrow 1$, we obtain many interesting new outcomes for the $p$-extended hypergeometric functions. Further, if we take both $k \rightarrow 1$ and $p \rightarrow 1$, then the obtained results reduce to the results analogous to the usual hypergeometric functions. This approach allows the related research work to be reported in further articles. Additionally, all the outcomes presented here are expected to find some applications in control theory and to the solutions of fractional-order systems, for instance, see [43-47] and the references cited therein. 
Funding

Not applicable.

Availability of data and materials

Not applicable.

\section{Declarations}

\section{Ethics approval and consent to participate}

Not applicable.

\section{Consent for publication}

Not applicable.

\section{Competing interests}

The authors declare that they have no competing interests.

\section{Authors' contributions}

The authors contributed equally in this article. They have all read and approved the final manuscript.

\section{Author details}

'Department of Mathematics, Faculty of Science, King Khalid University, Abha 61471, Saudi Arabia. ${ }^{2}$ Mathematics Department, Faculty of Science, South Valley University, Qena 83523, Egypt. ${ }^{3}$ Department of Mathematics, College of Sciences and Arts, ArRas, Qassim University, Buraydah, Kingdom of Saudi Arabia. ${ }^{4}$ Laboratory of Fundamental and Applied Mathematics of Oran (LMFAO), University of Oran 1, Oran, Algeria. ${ }^{5}$ College of Industrial Engineering, King Khalid University, Abha 62529, Saudi Arabia. ${ }^{6}$ Department of Mathematics Poornima College of Engineering ISI-6, RIICO Institutional Area, Jaipur, India.

\section{Publisher's Note}

Springer Nature remains neutral with regard to jurisdictional claims in published maps and institutional affiliations.

Received: 16 August 2021 Accepted: 29 September 2021 Published online: 14 October 2021

\section{References}

1. Agarwal, P., Agarwal, R.P., Ruzhansky, M.: Special Functions and Analysis of Differential Equations. Chapman and Hall, London (2020)

2. Agarwal, P., Baleanu, D., Chen, Y., Momani, S., Tenreiro Machado, J.A. (eds.): Fractional Calculus: ICFDA 20181 st edn. Springer Proceedings in Mathematics Statistics, vol. 303. Springer, Singapore (2019). https://doi.org/10.1007/978-981-15-0430-311

3. Agarwal, P., Dragomir, S., Jeli, M., Samet, B.: Advances in Mathematical Inequalities and Applications. Trends in Mathematics. Birkhäuser, Basel (2019)

4. Abdalla, M.: Special matrix functions: characteristics, achievements and future directions. Linear Multilinear Algebra $68,1-28(2020)$

5. Abdalla, M.: Fractional operators for the Wright hypergeometric matrix functions. Adv. Differ. Equ. 2020, Article ID 246 (2020)

6. Abdalla, M., Idris, S.A., Mekawy, I.: Some results on the extended hypergeometric matrix functions and related functions. J. Math. 2021, Article ID 2046726 (2021)

7. Abdalla, M., Bakhet, A.: Extended Gauss hypergeometric matrix functions. Iran. J. Sci. Technol. Trans. A, Sci. 42, 1465-1470 (2018)

8. Goyal, R., Momani, S., Agarwal, P., Rassias, M.: An extension of beta function by using Wimans function. Axioms 10 $187(2021)$

9. Agarwal, P., Chand, M., Purohit, S.D.: A note on generating functions involving the generalized Gauss hypergeometric functions. Nat. Acad. Sci. Lett. 37, 457-459 (2014)

10. Hidan, M., Abdalla, M.: A note on the Appell hypergeometric matrix function $F_{2}$. Math. Probl. Eng. 2020, Article ID $6058987(2020)$

11. Vivas-Cortez, M., Ali, M.A., Budak, H., Kalsoom, H., Agarwal, P.: Some new HermiteHadamard and related inequalities for convex functions via ( $p$; q)-integra. Entropy 23(7), 828 (2021)

12. Agarwal, P., Restrepo, J.E.: An extension by means of $\omega$-weighted classes of the generalized Riemann-Liouville k-fractional integral inequalities. J. Math. Inequal. 14, 35-46 (2020)

13. Diaz, R., Pariguan, E.: On hypergeometric functions and k-Pochhammer symbol. Divulg. Mat. 15, 179-192 (2007)

14. Srivastava, H.M., Choi, J.: Zeta and q-Zeta Functions and Associated Series and Integrals. Elsevier, Amsterdam (2012)

15. Ali, R.S., Mubeen, S., Ahmad, M.M.: A class of fractional integral operators with multi-index Mittag-Leffler $k$-function and Bessel $k$-function of first kind. J. Math. Comput. Sci. 22, 266-281 (2021)

16. Agarwal, P., Chand, M., Choi, J., Singh, G.: Certain fractional integrals and image formulas of generalized k-Bessel function. Commun. Korean Math. Soc. 33, 423-436 (2018)

17. Agarwal, P., Ntouyas, S., Jain, S., Chand, M., Singh, G.: Fractional kinetic equations involving generalized k-Bessel function via Sumudu transform. Alex. Eng. J. 57, 1937-1942 (2018)

18. Abdalla, M. Hidan, M.: Investigation of the k-analogue of Gauss hypergeometric functions constructed by the Hadamard product. Symmetry 13, 714 (2021) 
19. Mondal, S., Akel, M.: Differential equation and inequalities of the generalized k-Bessel functions. J. Inequal. Appl. 2018, $175(2018)$

20. Li, S., Dong, Y.: K-hypergeometric series solutions to one type of non-homogeneous k-hypergeometric equations. Symmetry 11, $262(2019)$

21. Yilmazer, R., Ali, K.: Discrete fractional solutions to the k-hypergeometric differential equation. Math. Methods Appl. Sci. 44, 7614-7621 (2021). https://doi.org/10.1002/mma.6460

22. Amsalu, H., Shimelis, B., Suthar, D.L.: Pathway fractional integral formulas involving S-function in the kerne. Math. Probl. Eng. 2020, Article ID 4236823 (2020)

23. Abdalla, M., Hidan, M., Boulaaras, S., Cherif, B.: Investigation of extended k-hypergeometric functions and associated fractional integrals. Math. Probl. Eng. 2021, Article ID 9924265 (2021)

24. Hidan, M., Boulaaras, S., Cherif, B., Abdalla, M.: Further results on the $(p ; k)$-analogue of hypergeometric functions associated with fractional calculus operators. Math. Probl. Eng. 2021, Article ID 5535962 (2021)

25. Mohamed, M.T.: Certain Functions and Sets of Polynomials and their Convergence Properties. PhD. Dissertation, Assiut University, Egypt (2003)

26. Debnath, L., Bhatta, D.: Integral Transforms and Their Applications, 3rd edn. Chapman and Hall, London (2015)

27. Kochubei, A., Luchko, Y.: Handbook of Fractional Calculus with Applications. Volume 1, Basic Theory. De Gruyter, Berlin (2019)

28. Gupta, V., Bhatt, M.: Some results of k-hypergeometric functions associated with integral transforms and fractional calculus. Int. J. Math. Sci. Comput. 5, 106-109 (2015)

29. Mubeen, S., Habibullah, G.M.: k-fractional integrals and application. Int. J. Contemp. Math. Sci. 7, 89-94 (2012)

30. Naz, S., Naeem, M.N.: On the generalization of k-fractional Hilfer-Katugampola derivative with Cauchy problem. Turk. J. Math. 45, 110-124 (2021)

31. Rahman, G., Mubeen, S., Nisar, K.: On generalized k-fractional derivative operator. AIMS Math. 5, 1936-1945 (2020)

32. Azam, M., Farid, G., Rehman, M.: Study of generalized type k-fractional derivatives. Adv. Differ. Equ. 2017, 249 (2017)

33. Kiryakova, V.: Unified approach to fractional calculus images of special functions a survey. Mathematics 8 , Article ID 136 (2020). https://doi.org/10.3390/math8122260

34. Zayed, M., Abul-Ez, M., Abdalla, M., Saad, N.: On the fractional order Rodrigues formula for the shifted Legendre-type matrix polynomials. Mathematics 8, Article ID 136 (2020)

35. Zayed, M., Hidan, M., Abdalla, M., Abul-Ez, M.: Fractional order of Legendre-type matrix polynomials. Adv. Differ. Equ. 2020, Article ID 506 (2020)

36. Mittal, E., Joshi, S.: Note on a k-generalised fractional derivative. Discrete Contin. Dyn. Syst., Ser. S 13, 797-804 (2020). https://doi.org/10.3934/dcdss.2020045

37. Gunerhan, H., Celik, E.: Analytical and approximate solutions of fractional partial differential algebraic equations. Appl. Math. Nonlinear Sci. 5, 109-120 (2020)

38. İlhan, E., Kıymaz, İ.O.: A generalization of truncated M-fractional derivative and applications to fractional differential equations. Appl. Math. Nonlinear Sci. 5, 171-188 (2020)

39. Yokus, A., Gulbahar, S.: Numerical solutions with linearization techniques of the fractional Harry Dym equation. Appl. Math. Nonlinear Sci. 4, 35-42 (2019)

40. Ziane, D., Cherif, M.H., Cattani, C., Belghaba, K.: Yang-Laplace decomposition method for nonlinear system of local fractional partial differential equations. Appl. Math. Nonlinear Sci. 4, 489-502 (2019)

41. Saigo, M., Maeda, N.: More generalization of fractional calculus, transform methods and special functions. In: Varna, vol. 96, p. 386-400. Bulgarian Academy of Sciences, Sofia (1998)

42. Kabra, S., Nagar, H., Nisar, K.S., Vyas, V.K.: Marichev-Saigo-Maeda fractional operators on generalized function $G_{\rho, \eta, \gamma}[a, z]$. Math. Eng. Sci. Aerosp. 11, 371-379 (2020)

43. Abdalla, M., Akel, M.: Computation of Fourier transform representations involving the generalized Bessel matrix polynomials. Adv. Differ. Equ. 2021, Article ID 418 (2021)

44. Matsubara-heo, S.J.: On Mellin-Barnes integral representations for GKZ hypergeometric functions. Kyushu J. Math. 74, 109-125 (2020)

45. Guefaifia, R., Boulaaras, S.M., El-Sayed, A.A., Abdalla, M., Cherif, B.: On existence of sequences of weak solutions of fractional systems with Lipschitz nonlinearity. J. Funct. Spaces 2021, Article ID 5510387 (2021)

46. Kamache, F., Boulaaras, S.M., Guefaifia, R., Chung, N.T., Cherif, B., Abdalla, M.: On existence of multiplicity of weak solutions for a new class of nonlinear fractional boundary value systems via variational approach. J. Funct. Spaces 2021, Article ID 5544740 (2021)

47. Boulaaras, S., Choucha, A., Cherif, B., Alharbi, A., Abdalla, M.: Blow up of solutions for a system of two singular nonlocal viscoelastic equations with damping, general source terms and a wide class of relaxation functions. AIMS Math. 6(5), 4664-4676 (2021) 